\title{
Stimulus Site and Modality Dependence of Functional Activity within the Human Spinal Cord
}

\author{
Jonathan C. W. Brooks, ${ }^{1}$ Yazhuo Kong, ${ }^{1}$ Michael C. Lee, ${ }^{2}$ Catherine E. Warnaby, ${ }^{1}$ Vishvarani Wanigasekera, ${ }^{1}$ \\ Mark Jenkinson, ${ }^{1}$ and Irene Tracey ${ }^{1}$ \\ ${ }^{1}$ Centre for Functional Magnetic Resonance Imaging of the Brain, Nuffield Department of Clinical Neurosciences, Nuffield Division of Anaesthetics, \\ University of Oxford, Oxford OX3 9DU, United Kingdom, and ²Department of Medicine, Division of Anaesthesia, University of Cambridge, Cambridge CB2 \\ 2QQ, United Kingdom
}

Chronic pain is thought to arise because of maladaptive changes occurring within the peripheral nervous system and CNS. The transition from acute to chronic pain is known to involve the spinal cord (Woolf and Salter, 2000). Therefore, to investigate altered human spinal cord function and translate results obtained from other species, a noninvasive neuroimaging technique is desirable. We have investigated the functional response in the cervical spinal cord of 18 healthy human subjects (aged 22-40 years) to noxious thermal and non-noxious tactile stimulation of the left and right forearms. Physiological noise, which is a significant source of signal variability in the spinal cord, was accounted for in the general linear model. Group analysis, performed using a mixed-effects model, revealed distinct regions of activity that were dependent on both the side and the type of stimulation. In particular, thermal stimulation on the medial aspect of the wrist produced activity within the C6/C5 segment ipsilateral to the side of stimulation. Similar to data recorded in animals (Fitzgerald, 1982), painful thermal stimuli produced increased ipsilateral and decreased contralateral blood flow, which may reflect, respectively, excitatory and inhibitory processes. Nonpainful punctate stimulation of the thenar eminence provoked more diffuse activity but was still ipsilateral to the side of stimulation. These results present the first noninvasive evidence for a lateralized response to noxious and non-noxious stimuli in the human spinal cord. The development of these techniques opens the path to understanding, at a subjectspecific level, central sensitization processes that contribute to chronic pain states.

\section{Introduction}

Functional imaging studies have revealed that pain sensation is associated with activity across widespread cortical networks (Jones et al., 1991; Talbot et al., 1991). However, activation of peripheral nociceptors is not a prerequisite for feeling pain (Merskey and Bogduk, 1994; Rainville et al., 1997; Raij et al., 2005). This dichotomy presents problems when attempting to understand the origins of pain and how psychological and nociceptive processes interact. The amount of pain experienced after noxious stimulation depends on several factors, such as cortical amplification during directed attention (Miron et al., 1989), subcortical modulation by pronociceptive and antinociceptive networks (Fields, 2004), and spinal amplification caused by maladaptive changes such as central sensitization (Latremoliere and Woolf, 2009). The degree to which each of these contributes to the perception of pain, particularly in disease states, is unknown. The

Received May 19, 2011; revised March 6, 2012; accepted March 10, 2012.

Author contributions: J.C.W.B., M.C.L., C.E.W., V.W., and I.T. designed research; J.C.W.B., Y.K., M.C.L., C.E.W., and V.W. performed research; J.C.W.B., Y.K., and M.J. analyzed data; J.C.W.B., M.J., and I.T. wrote the paper.

This work was supported by grants from the Medical Research Council of Great Britain and Northern Ireland (J.C.W.B, Y.K., and M.C.H.L.), Wellcome Trust (V.W. and I.T.), Biotechnology and Biological Sciences Research Council (M.J.), and the National Institute for Health Research Biomedical Research Centre Programme (C.E.W.).

The authors declare no competing financial interests.

Correspondence should be addressed to Jonathan C. W. Brooks, FMRIB Centre, Nuffield Department of Clinical Neurosciences, John Radcliffe Hospital, Oxford OX3 9DU, United Kingdom; E-mail: jon@fmrib.ox.ac.uk.

DOI:10.1523/JNEUROSCI.2543-11.2012

Copyright $\odot 2012$ the authors $\quad 0270-6474 / 12 / 326231-09 \$ 15.00 / 0$ development of noninvasive imaging techniques to record spinal activity would provide a crucial first step toward disentangling these processes and would offer insight into patterns of cortical activity observed in healthy controls and pain patients.

Imaging techniques for assessing nociceptive spinal cord activity in animals initially focused on autoradiographic techniques (Coghill et al., 1991; Porro et al., 1991) and, more recently, on blood flow-sensitive functional magnetic resonance imaging (fMRI) (Porszasz et al., 1997; Lilja et al., 2006; Zhao et al., 2008; Cohen-Adad et al., 2009). These animal data demonstrated a lateralized response to noxious stimuli-with the greatest activity ipsilateral to the side being stimulated. Spinal responses to painful stimuli have been recorded in humans using fMRI (Lawrence et al., 2008a; Summers et al., 2010) and activity observed bilaterally. Reasons for this discrepancy include failure to account for physiological noise affecting spinal cord images (Stroman, 2006; Brooks et al., 2008; Piché et al., 2009), the small size of the spinal cord, and the pressing need to develop methods for group analysis of such data. To date, a single fMRI study (Eippert et al., 2009) using unilateral stimulation and physiological noise correction has demonstrated ipsilateral spinal cord activity following painful thermal (T) stimulation.

To address limitations in previous experiments and examine spinal responses in greater detail, we have applied carefully controlled and calibrated stimuli to separate dermatomes on both sides of the body. In particular, we recorded spinal activity using blood oxygenation level-dependent (BOLD)-sensitive imaging 
(Bouwman et al., 2008; Cohen-Adad et al., 2010) in response to phasic heat stimuli applied with a thermal resistor to produce mild to moderate pain and punctate $(\mathrm{P})$ stimulation using a von Frey hair to produce a sharp (nociceptive but nonpainful) sensation. On the basis of data recorded in other species, we predicted that spinal BOLD activity would occur primarily in the dorsal horn of the spinal cord ipsilateral to the site of stimulation, and the segmental distribution of activity would depend on the specific dermatomes stimulated. To facilitate these comparisons, we have developed methods necessary to perform group analysis, and we present data from the human spinal cord that for the first time is based on results from mixed-effects analysis.

\section{Materials and Methods}

Eighteen healthy subjects (seven women) aged between 22 and 40 years (mean, 28.4 years; SD, 2.6 years) were recruited and screened for participation in the experiment. All subjects provided written consent to the experimental procedures (World Medical Association, 1996). In addition to the normal exclusion criteria for fMRI experiments (e.g., history of brain trauma, presence of neurological or psychiatric disease), subjects with a history of long-term analgesic use were excluded from the study. On the basis of a handedness questionnaire (Oldfield, 1971), one subject was assessed to be left handed but was included in the study because there is no evidence of spinal cord changes associated with handedness. Subjects were instructed to abstain from analgesic medication on the day of the study. Scanning was performed using a 3 tesla MR system (Siemens) with the standard 12-channel head coil, and anterior/posterior neck array (four channels). During functional imaging only the neck elements were selected for signal reception. Stimulation consisted of T and P stimulation of the right and left wrist/hand. Punctate stimuli were delivered using $60 \mathrm{~g}$ of von Frey hair (Stoelting) to the thenar eminence (base of thumb), while painful thermal stimuli were delivered to the medial aspect of the volar forearm (wrist) using an in-house built thermal stimulator (Brooks et al., 2005). Thus, stimuli were nominally targeted to the C6 (thumb) and C8 (wrist area) dermatomes of both arms. After positioning in the scanner, each subject's pain threshold was assessed using a staircase technique and a numerical rating scale (with " 0 " equal to "no pain" and "10" equal to "worst pain imaginable"). For each subject, the temperature of the 3-s-long stimuli was adjusted to produce a stable pain rating between 5 and 6 .

After the acquisition of localizer images, T1-weighted sagittal spin echo data were acquired for functional image prescription. fMRI data were acquired with a gradient echo (GRE)-echo planar imaging (EPI) sequence and the following parameters: $\mathrm{TE} / \mathrm{TR}=39 / 1000 \mathrm{~ms}$, flip angle $68^{\circ}$, matrix $96 \times 96$, field of view $128 \mathrm{~mm}$, and parallel acceleration $($ Factor $=2$, GRAPPA reconstruction algorithm) in the posterioranterior direction to minimize susceptibility-induced compression of the cord. Nine axial-oblique slices ( $4 \mathrm{~mm}$ thick) were prescribed perpendicularly to the long axis of the spinal cord and were located from the vertebral body of C4 down to T1 (see Fig. 5). The interslice gap was adjusted for each subject such that slices were aligned with the midpoints of the vertebral bodies and intervertebral discs.

Stimulation of the four sites was performed with a pseudorandomized order during a single fMRI run lasting $42 \mathrm{~min}$ and $44 \mathrm{~s}$, equivalent to 2564 volumes. Thermal stimulation was controlled using in-house developed software that was triggered by the scanner. Punctate stimuli lasting $1 \mathrm{~s}$ were delivered by an experimenter and timed by computer-controlled auditory instructions presented through headphones. To avoid skin sensitization, stimulation alternated between modality (T/P). The side of stimulation was randomized and the interstimulus interval jittered to reduce effects of anticipation. After each stimulus, following a delay of $12 \mathrm{~s}$, subjects rated its intensity (thermal) or sharpness (punctate). Ratings were provided with an MR-compatible potentiometer held in the right hand, which controlled a visual analog scale (VAS) visible to subjects in the scanner with the verbal descriptors "not painful" " not sharp" and "intolerable" used as anchors at the bottom and top ends of scales, respectively. During scanning, cardiac and respiratory waveforms were recorded using a pulse-oximeter and pneu- matic respiratory bellows and were logged along with volume triggers from the scanner on a BIOPAC MP150 (Biopac Systems).

Functional data were processed on a per-slice basis using tools from the Centre for Functional MRI of the Brain Software Library (FSL; http:// www.fmrib.ox.ac.uk/fsl). Data were motion corrected using a custom 2D routine using FLIRT (Jenkinson et al., 2002), and transformations were limited to translations along the $x$ - and $y$-directions. Rotations and translations in the $z$-direction were assumed to be minimal, which was confirmed by visual inspection following motion correction (Cohen-Adad et al., 2007). Subsequently, data were smoothed with a $3 \mathrm{~mm}$ FWHM kernel and were high-pass filtered with the cutoff set to $300 \mathrm{~s}$. Before model estimation, data were masked to include only tissue contained within the spinal cord. The presence of activation was assessed using a general linear model (GLM) (FEAT, version 5.98) (Woolrich et al., 2004) incorporating a physiological noise model (PNM) (Brooks et al., 2008). The model included regressors for the four different stimuli-thermal pain right (RT) and left (LT) and punctate stimulation right (RP) and left (LP)and four regressors for the four rating periods associated with each stimulus. Contrasts were defined for the four different stimuli versus baseline and for the difference between right- and left-sided stimulation (within modality) (i.e., RP > LP, LP > RP, RT > LT, and LT > RT). Last, a contrast estimating the average of the four rating periods (a sensorimotor task) was defined.

To assess the impact of the physiological noise model on estimating the response to each of the four stimuli, the signal time course around each stimulus was extracted, and the mean peristimulus plot was calculated along with the fitted hemodynamic response. Signal time course extraction used the location of the peak voxel (highest $Z$-score) identified by the group analysis and transformed back into each subject's original fMRI data. The improvement in modeling can be more easily assessed by comparing the residual sum of squares (RSS) after applying different models. To measure this, data for all 18 subjects were pooled, and the RSS was computed for all voxels falling within a spinal cord mask. The RSS was calculated for the raw data (no model), the model including only the experimental design ("basic"), the basic model plus 32 physiological regressors (basic + PNM), and the basic model plus PNM and a regressor derived from voxels in the CSF space surrounding the cord (basic + PNM + CSF) - the chosen model for activation estimation. Reduction in RSS, which reflects improved modeling of the data, was assessed by computing the percentage difference between the RSS for the raw data and each of the three models, and the mean over the group was calculated.

After model estimation, per-slice group analysis was performed using the following steps: (1) a single subject with minimal curvature of the spinal cord was identified as the "standard" spinal cord, and the outline of the cord was drawn manually to produce a standard cord mask; (2) cord masks were drawn for the remaining 17 subjects; and (3) cord masks were coregistered to the standard cord mask using a 4 degrees of freedom transformation (translation and scaling in the $x$ and $y$ directions). The resultant transformation matrices were then applied to the functional data to place all subjects in a common space for group model estimation (see Fig. 1). Group activation was assessed using a mixed-effects model, and results are reported with a "corrected" threshold of $p<0.003$. Unlike brain imaging, in which numerous approaches to adjusting $p$ values to control familywise error rates have been developed, these techniques are generally not suitable for studying extremely small structures such as the cord (e.g., Gaussian random field theory) (Worsley et al., 1992). The adopted threshold reflects the low number of voxels present in the spinal cord mask (see Discussion) and is based on dividing the adopted significance level ( $p=0.05$ ) by the number of acquired slices multiplied by the number of hemicords (i.e., 9 slices $\times 2$ hemicords $=18$ ), giving $p=$ 0.003 . Note that uncorrected $p$ values are frequently used when assessing the significance of cord activation (Moffitt et al., 2005; Brooks et al., 2008; Eippert et al., 2009; Cohen-Adad et al., 2010; Stroman et al., 2011).

An alternative to parametric testing within the framework of the GLM is nonparametric testing with permutation tests (Nichols and Holmes, 2002). The significance of spinal cord activation was assessed by using the permutation test in FSL (RANDOMISE; Centre for Functional MRI of the Brain). For each slice, the maximum $t$-statistic across all voxels in this 
Table 1. Summary statistics for applied temperatures and online psychophysical ratings obtained from subjects during scanning

\begin{tabular}{|c|c|c|c|c|c|c|}
\hline & \multirow{2}{*}{\multicolumn{2}{|c|}{$\begin{array}{l}\text { Punctate } \\
\text { Sharpness } \\
(60 \mathrm{~g} \text { of von Frey })^{a}\end{array}$}} & \multicolumn{4}{|l|}{ Thermal } \\
\hline & & & \multicolumn{2}{|c|}{$\begin{array}{l}\text { Applied temperature } \\
\left({ }^{\circ} \mathrm{C}\right)\end{array}$} & \multicolumn{2}{|c|}{$\begin{array}{l}\text { Pain rating } \\
\text { (VAS) }\end{array}$} \\
\hline & Right & Left & Right & Left & Right & Left \\
\hline Mean & 2.1 & 1.9 & 52.3 & 51.3 & 3.6 & 3.6 \\
\hline SD & 0.9 & 0.8 & 2.7 & 3.0 & 1.1 & 1.0 \\
\hline Lower $95 \% \mathrm{Cl}$ & 1.7 & 1.6 & 51.0 & 49.8 & 3.0 & 3.1 \\
\hline Upper 95\% Cl & 2.6 & 2.3 & 53.6 & 52.7 & 4.1 & 4.1 \\
\hline Test for normality ${ }^{b}$ & Yes & Yes & Yes & Yes & Yes & Yes \\
\hline Paired $t$ test & n.s. & & $p=0.004$ & $p=0.004$ & n.s. & \\
\hline
\end{tabular}

n.s., Not significant.

${ }^{a}$ von Frey nylon filament $(60 \mathrm{~g})$.

${ }^{b} D^{\prime}$ Agostino and Pearson omnibus normality test.

slice was obtained by randomly inverting the sign of the data (i.e., permuting the sign of the weighting vector used to specify the group) and repeating this 5000 times. For each permutation, the voxelwise $t$-statistic was recorded to finally form the permutation distribution of the maximum $t$-statistic within the slice. The resultant statistical maps present conservative estimates of significance of activity assessed across the group and are inherently adjusted for multiple comparisons by using the maximum $t$-statistic.

To understand the nature of group differences (e.g., RT $>$ LT), the coordinate representing the peak of activation from the group map was transformed back to each subject's space, and the BOLD percentage signal change for the corresponding first-level regressors was computed and plotted. To further interrogate patterns of activation seen in the grouplevel maps, region of interest (ROI) analyses were performed. To extract BOLD signal parameter estimates, the right and left hemicords were manually identified on the average of the functional images for each subject. Care was taken not to include voxels around the periphery of the cord, with the exception of voxels at the dorsal surface of the cord (see Fig. 1). To investigate the laterality of BOLD signal changes, repeatedmeasures ANOVA was performed in PASW 18 software (SPSS) with within-subject factors of site (left- or right-hand stimulation), side (ipsilateral or contralateral), and level (slices 1-8). Laterality effects were thus indicated by a main effect of side (e.g., ipsilateral activity was greater than activity contralateral to the site of stimulation) and an influence of segmental input to the cord reflected by a side-by-level interaction. To further investigate patterns of laterality, pairs of slices (e.g., slices 1 and 2, 3 and 4) were averaged together and subjected to a repeated-measures ANOVA with within-subject factors of site (left- or right-hand stimulation) and side (ipsilateral or contralateral). Significance was assessed at a level of $p<0.05$, which differs from the voxel-level threshold because the ANOVA constitutes a single statistical test. The rationale for averaging pairs of slices was to account for anatomical heterogeneity in the segmental input to cord (i.e., allowing for BOLD signal changes on adjacent slices).

\section{Results}

\section{Psychophysics}

The mean (SD) temperature of the applied thermal stimulus required to produce a pain rating of 5 of 10 (assessed before scanning) on the right and left wrists was $52.3^{\circ} \mathrm{C}\left(2.7^{\circ} \mathrm{C}\right)$ and $51.3^{\circ} \mathrm{C}$ $\left(3.0^{\circ} \mathrm{C}\right)$, respectively (Table 1$)$. These temperatures were significantly different $(p<0.05$, paired $t$ test), in agreement with an earlier study on the effect of handedness on perceived pain intensity (Sarlani et al., 2003). However, average pain intensity ratings recorded during scanning were not significantly different [right, 3.6 (1.1); left, 3.6 (1.0); $p=0.52$, paired $t$ test]. Considering the punctate stimulus applied to the base of the thumb, no subject reported the $60 \mathrm{~g}$ of von Frey hair as painful, and their average sharpness ratings (Lee et al., 2008) recorded online for right- and
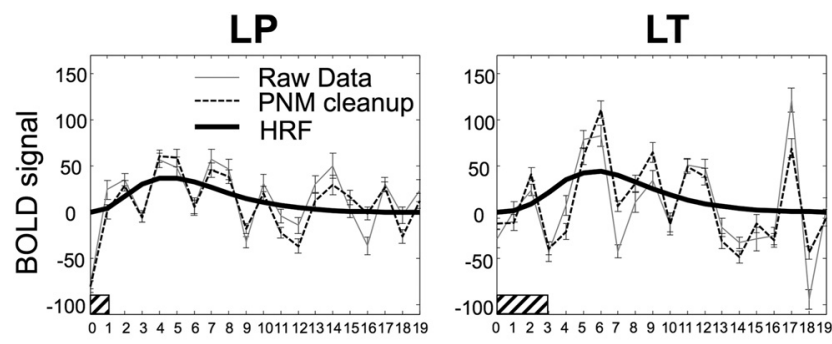

RP

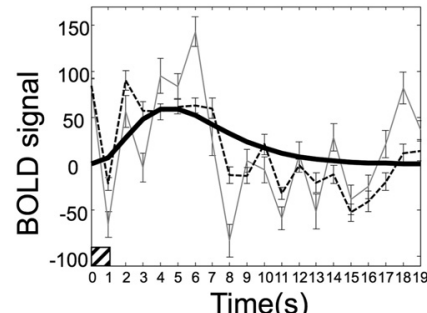

RT

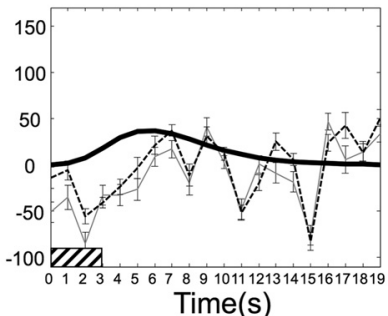

Figure 1. Group average $(N=18)$ peristimulus plots for each of the four applied stimuli (RP $L P, R T$, and LT) taken from the peak voxel for each contrast in the group analysis. For each of the four peristimulus plots, the raw data are shown by the light solid line, and the time course after "PNM cleanup" is shown by the light dashed line. Error bars represent SEs. In general, the data after cleanup have fewer large jumps and look smoother, reflecting the reduction in data variation. The heavy solid line is the fitted hemodynamic response curve to the data after cleanup, the filled bar at the origin indicates the timing and duration of the applied stimulus, and HRF $=$ haemodynamic response function.

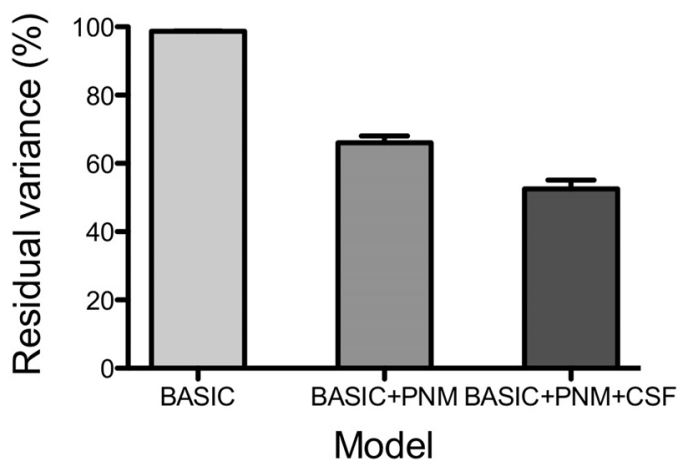

Figure 2. Average variance reduction for three applied models (basic, basic + PNM, and basic + PNM + (SF) measured from voxels in the spinal cord. The percentage reduction in variance is relative to the baseline variance of the raw data and is expressed as a percentage of the nominal value (100\%). Applying the basic design (experimental design only) reduced the variance by $<2 \%$, applying a PNM-reduced variance by a further $33 \%$, and including a CSF regressor in addition to the PNM reduced the overall variance by $47.5 \%$. The basic + PNM + CSF model was used to estimate activation in this study.

left-hand stimulation were $2.1(0.9)$ and 1.9 (0.8), respectively. The difference between sharpness ratings was not significantly different $(p=0.24$, paired $t$ test $)$.

\section{Functional imaging data}

Of the nine slices imaged, the bottom slice typically suffered from severe ghosting and low signal-to-noise ratio; therefore, only the topmost eight slices were analyzed. The impact of physiological noise modeling can be assessed by inspecting Figures 1 and 2. The group average peristimulus plots for the peak voxel from each of the four applied stimuli are shown in Figure 1 and demonstrates that small variations in the baseline (after removing physiological noise) contribute to improved estimation of the hemodynamic response function. However, the improvement in modeling is clearest when comparing the variance of the residuals (RSS) ob- 


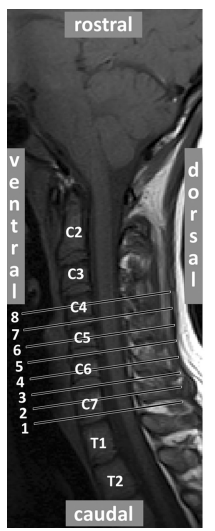

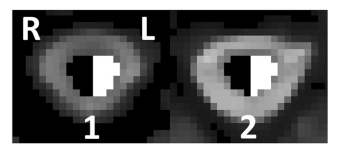

$p=0.031$
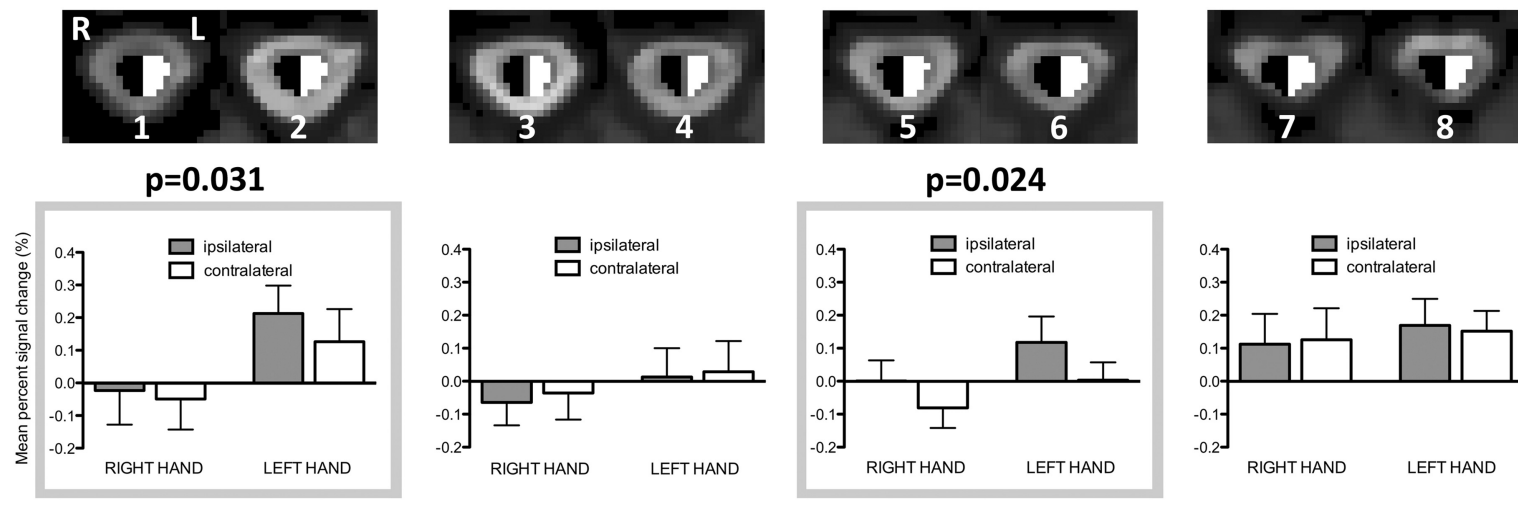

$p=0.024$
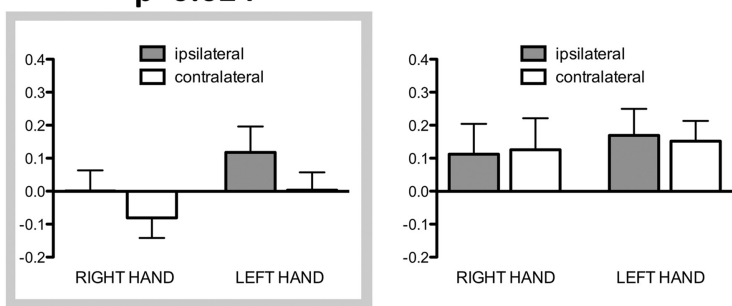

Figure 3. ROI analysis of spinal cord activation in response to punctate stimulation. BOLD percentage signal change was extracted from hand-drawn ROIs covering the dorsal and ventral regions of the right (black) and left (white) hemicords. The location of the relevant slices is shown on a sagittal section spanning from T1/C7 to C4 (i.e., covering the dorsal roots corresponding to dermatomes stimulated in this experiment). Averaged signal from adjacent slices was plotted (bar plots) for each stimulus location and each R0I, where bar shading corresponds to ipsilateral (filled bars) and contralateral (open bars) and thus refers to different sides of the cord, depending on the site of stimulation. A main effect of laterality was found for slices 1 and 2 and for slices 5 and 6 (repeated-measures ANOVA, $p<0.05$ ).

tained from different applied models (Fig. 2), averaged over the whole cord volume scanned. The basic model reduced residuals by a small amount (typically 1-2\%), whereas including a physiological noise model reduced this by $34 \%$, and including a regressor with signal variation recorded from the CSF surrounding the cord reduced signal variation by $47 \%$.

\section{ROI analysis}

To examine whether consistent patterns of activation (e.g., a lateralized BOLD response) were present at different spinal cord levels during punctate stimulation, we performed ROI analyses. The anatomical location of functional imaging slices is shown superimposed on a sagittal section taken through the cervical cord; right (black) and left (white) hemicord masks are shown for the corresponding slices (Fig. 3). The repeated-measures ANOVA with three factors (side, site, and level) demonstrated a main effect of side (ipsilateral greater than contralateral; $p=$ 0.034 ) and a trend toward significance for the interaction (side by level; $p=0.081$ ). To further investigate the nature of the main effect of side, activity within adjacent slices was averaged (see Materials and Methods), and laterality of activation for each of the resultant four cord levels was assessed using repeatedmeasures ANOVA. A main effect of laterality (e.g., greater BOLD activity ipsilateral to the side of stimulation) was found for slices 1 and 2 and slices 5 and 6 . The result was interrogated further by plotting activity (ipsilateral/contralateral) for each hemicord and for each condition (right or left hand stimulation) (Fig. 3, bar plots). It can be seen that activity was significantly greater ipsilateral (filled bar) than contralateral (open bar), for both right- and left-hand stimulation. Note that the average activity for righthand stimulation appears to be somewhat lower than for lefthand stimulation.

\section{Group analysis}

Group functional data are presented in Figure 4. At the chosen threshold ( $p<0.003$, corrected) activity was found for all contrasts, though most notably for punctate stimulation of the leftthumb and the right-hand motor task. For punctate stimulation of the right thumb, the cluster with peak $Z$-score was located ipsilateral and at the level of $\mathrm{C} 5 / \mathrm{C} 4$. For left-hand stimulation, the activity was more diffuse (at T1/C7, C6, and C4) but was again primarily located in the ipsilateral hemicord. Activity following thermal stimulation at the right wrist was located ipsilateral at the dorsal C6/C5 level, whereas for left-wrist stimulation active voxels were observed bilaterally at C6/C5 (though the peak voxel was again ipsilateral to the stimulus side) and at C7/C6 (midline, ventral), C5/C4(contralateral), and C4 (ipsilateral). The largest response resulted from the motor/sensory component of the rating task. In six of the eight spinal levels recorded, the focus of activity (largest cluster or peak voxel) was ipsilateral to the hand holding the VAS rating device and typically extended from dorsal to ventral regions. Activity located near the dorsal surface typically spanned both sides of the cord. The corresponding results obtained using nonparametric permutation testing are shown in Figure 5. Results are corrected for multiple comparisons, and a threshold of $p<0.05$ was applied. The patterns of activity broadly agree with the data obtained with the uncorrected threshold (parametric model). Notably, activation in response to punctate stimulation was ipsilateral to the side of stimulation, which was also true for left thermal stimulation. Although no activity was found for right thermal stimulation, the pattern of activity in response to the sensorimotor (rating) task is in excellent agreement with the uncorrected data.

\section{Group differences}

Within each modality (thermal or punctate), the group differences between activation patterns produced following stimulation of the left and right sides of the body were calculated. The group differences between left and right punctate stimuli are shown in Figure 6. In common with the general pattern of activation for the initial group analysis, the difference contrasts revealed activity that was primarily ipsilateral to the side of stimulation. For RP $>$ LP, with the exception of two voxels located on the cord/CSF boundary on the dorsal side, active voxels were observed in the right (ipsilateral) ventral hemicord at the C5/C4 and C4 level. For the LP > RP contrast, two clusters of activity were observed in the left (ipsilateral) hemicord, extending from dorsal to ventral regions (T1/C7 level) and more ventrally at (C6). The origin of these group differences was investigated by estimating the BOLD percentage signal change for each subject and each contrast (RP and LP) at the peak voxel from the group difference maps. The average BOLD signal change and SE are shown at the activated spinal cord levels; see Figure 6, which reveals that, for some spinal levels, the voxelwise 


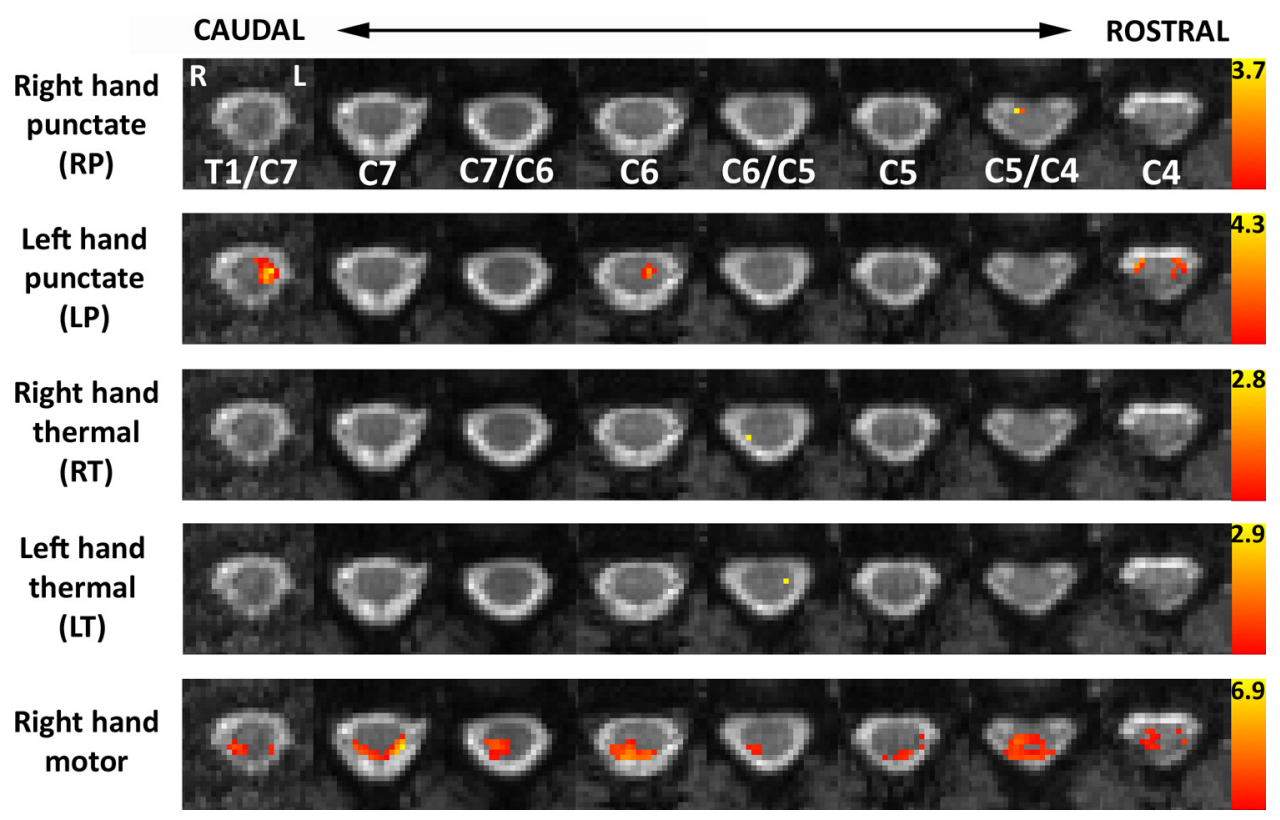

Figure 4. Mixed-effects group results for punctate/thermal stimuli and rating task. Activation data, thresholded at "corrected" $p<0.003$ (corresponding to $Z>2.75$ ), are superimposed on the corresponding slices of the average EPI data from the standard cord. Activation was found for all contrasts and tended to be located ipsilaterally to the side of thermal or punctate stimulation. Activity during the four separate rating periods was averaged and constituted a right-hand sensory/motor task. Activity was located primarily within the ipsilateral hemicord and extended from dorsal (sensory) to ventral (motor) areas. Note that activity on the CSF/cord boundary was found exclusively on the dorsal cord surface and tended to be bilateral. Data are shown in radiological format (left side of cord on right side of each slice), and the level of each slice is shown relative to the vertebral body to which it is opposite (or at the midpoint between two vertebrae if that is where it lies).

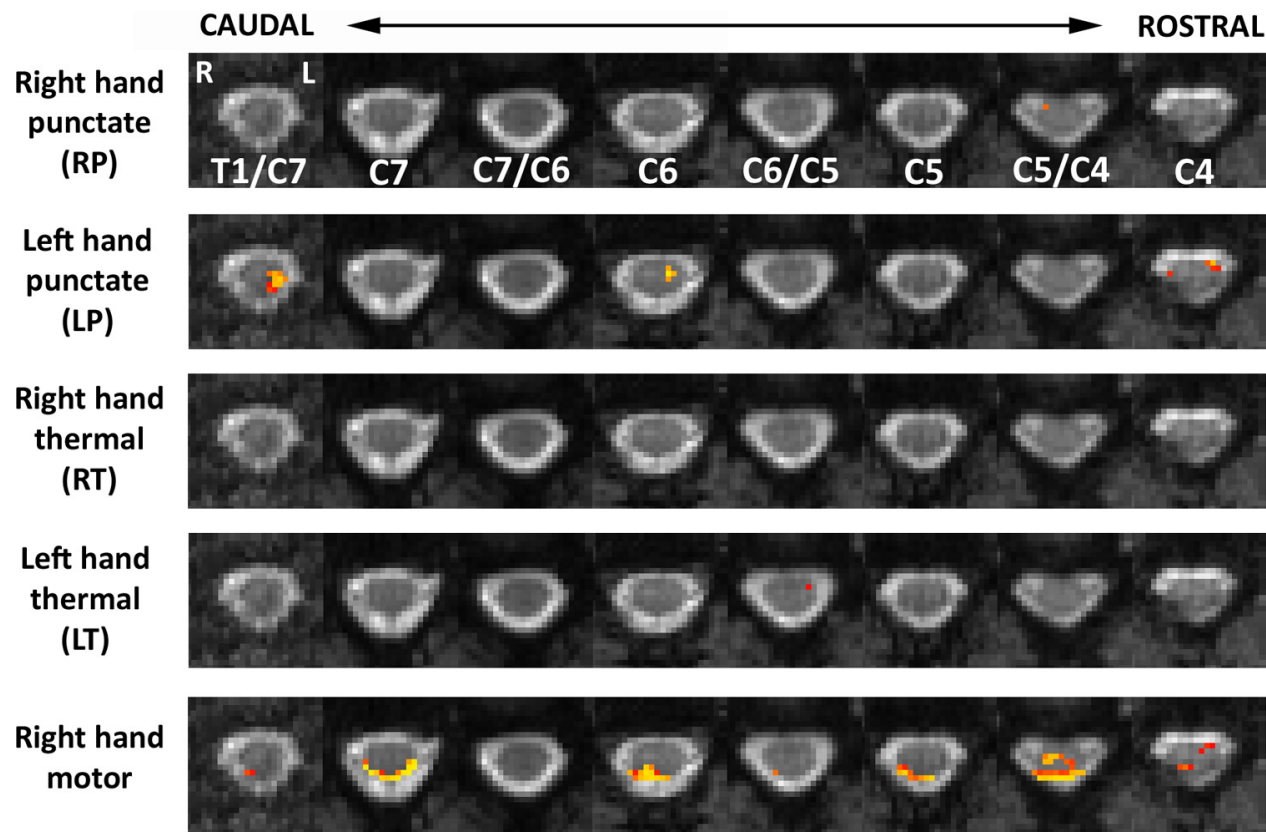

Figure 5. Group results for punctate/thermal stimuli and rating task, subjected to a nonparametric permutation testing of the maximum $t$-statistic for each voxel and corrected for multiple voxelwise comparisons $(p<0.05)$. The resultant activation data are superimposed on the corresponding slice of the average EPI data from the standard cord. The location of activation for each of the group contrasts, following the robust correction of multiple voxel-based comparisons, broadly agrees with results obtained with parametric thresholding ("corrected," $p<0.003$; see Fig. 4), with the exception of right thermal stimulation during which no activation was statistically significant. Activation was primarily ipsilateral to the side of stimulation (RP, LP, and LT). The rating (right-hand sensorimotor) task activated the dorsal cord bilaterally (though primarily ipsilaterally) and the ventral cord primarily ipsilaterally (C5/C4).

differences resulted from positive BOLD signal during one condition and negative BOLD signal during the other condition.

For thermal stimulation (Fig. 7), the significant group differences RT $>$ LT and LT $>$ RT were each found at the single spinal cord levels $\mathrm{C} 6$ and $\mathrm{C} 6 / \mathrm{C} 5$, respectively. For each of the computed contrasts, the significant voxels were located ipsilateral to the side stimulated. As for punctate stimulation, the nature of these differences was investigated by computing the mean \pm SE of BOLD percentage signal change for each of the contrasts (RT and LT). The data demonstrate that group differences between thermal responses were driven by positive BOLD signal in one condition and negative BOLD signal in the other. The result suggests that during thermal stimulation of either hand, activation was positive in the hemicord ipsilateral to the 


\section{right punctate $>$ left punctate}
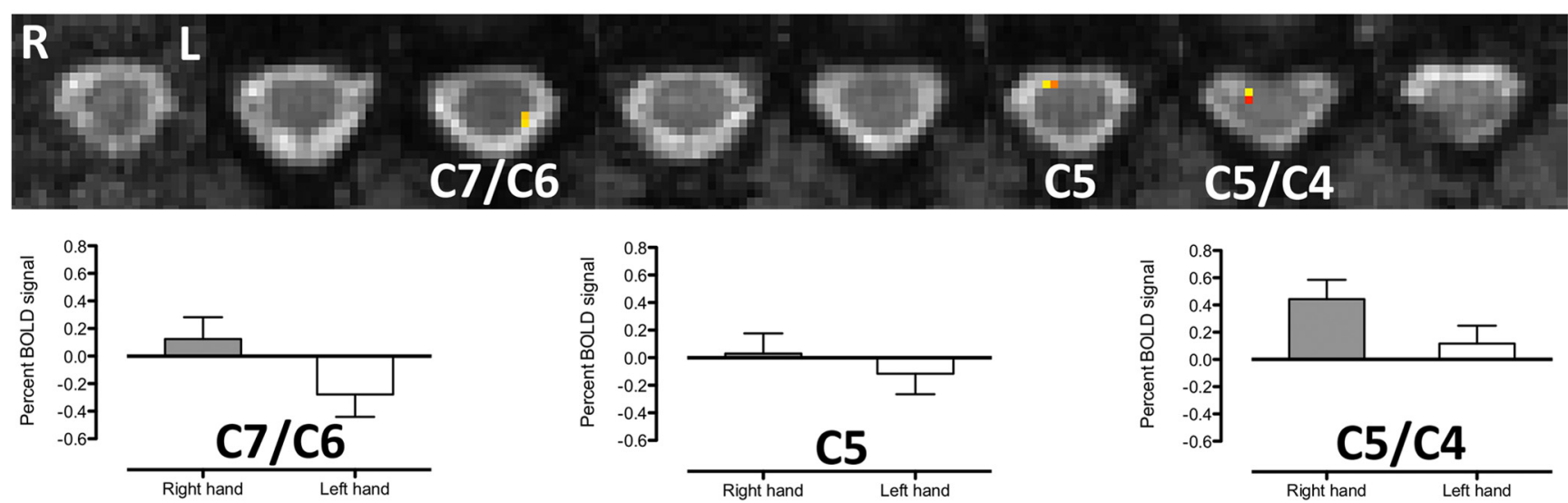

\section{left punctate $>$ right punctate}
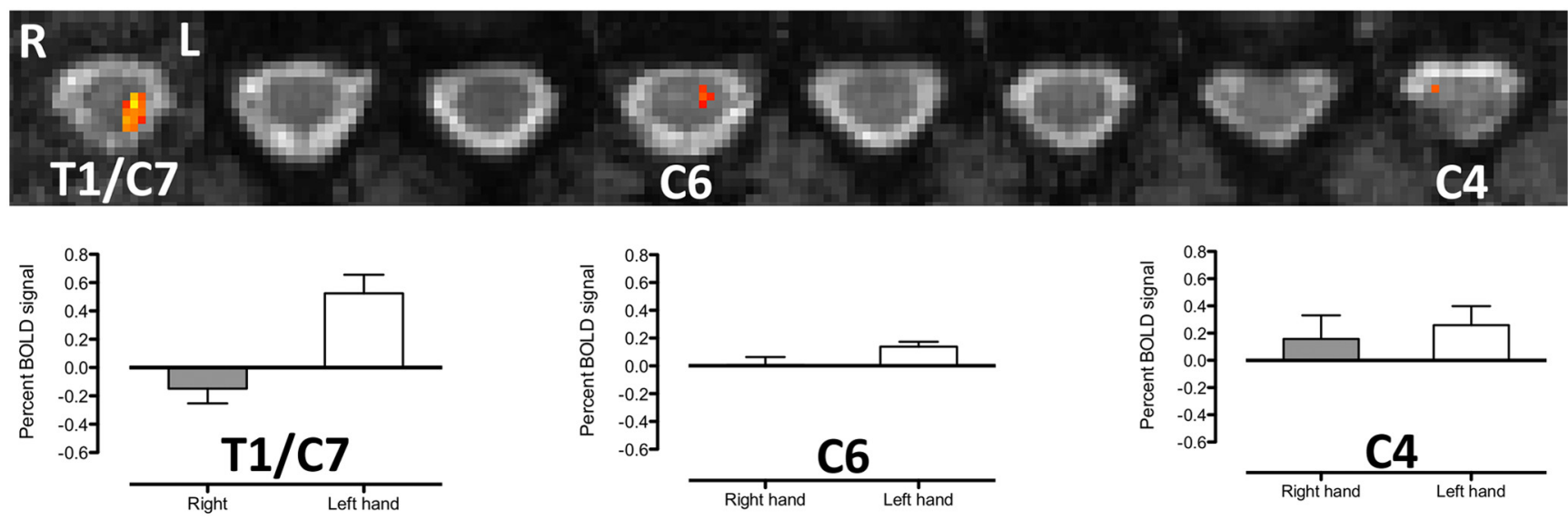

Figure 6. Mixed-effects group differences between LP and RP stimulation. Group differences between the individual contrasts were broadly consistent with the ipsilateral pattern of activity for each separate contrast. The nature of the differences was investigated by computing the BOLD percentage signal change for the peak voxel (highest Z-score) for each slice, shown in the corresponding bar plots for the activated spinal cord levels. The statistical threshold was $p<0.01$ (uncorrected).

side stimulated and negative in the side contralateral to the stimulus.

\section{Discussion}

This study used BOLD-based functional imaging and a novel approach to group analysis to record spinal cord responses to nociceptive stimulation in humans. To increase the statistical power to detect activation, we included in the general linear model a set of regressors designed to account for cardiac and respiratory noise and their interaction and for low-frequency signal components. Furthermore, we developed techniques for coregistration of single-subject data to a standard spinal cord for group averaging of data. In response to painful thermal stimulation of the right and left wrists, we observed activity that was ipsilateral to the side of stimulation. Our experimental design permitted direct comparison of stimuli delivered to opposite sides of the body, which confirmed the ipsilateral cord response. Intriguingly, this group difference arose because of increased ipsilateral and decreased contralateral spinal activity during painful thermal stimulation. Similarly, punctate stimulation of the thenar eminence also produced activity that was ipsilateral to the side of stimulation, which was confirmed with ROI analysis. In terms of the rostrocaudal level of spinal activity, no clear pattern of activation emerged in response to stimulation of different dermatomes.

By delivering punctate and thermal stimuli to the C6 and C8 dermatomes, respectively, we investigated whether activity could be located to the corresponding segmental level (i.e., whether a spinal somatotopic representation of the body was observable). In practice, activity in response to nonpainful punctate stimulation had greater rostrocaudal extent than to thermal stimuli (Fig. 4). In response to brief, repetitive ( $4 \mathrm{~ms}$ ) nociceptive laser stimulation of the dorsum of the left hand (C6/C7 dermatomes), extensive bilateral activity extending from $\mathrm{C} 4$ to $\mathrm{C} 7$ has been reported (Summers et al., 2010), with brief laser stimuli apparently activating more ventral cord regions, suggesting involvement of the motor neuron pool in the measured response. Bilateral activity extending over several segments $(\mathrm{C} 4-\mathrm{C} 7)$ has previously been observed following non-nociceptive vibratory stimulation (targeting large-diameter $\mathrm{A} \beta$ afferents) of the arm (Lawrence et al., 2008b). Furthermore, in the anesthetized rat, electrical stimulation of the plantar surface of a single forepaw produced activity that extended from C2 to T1 (Zhao et al., 2009). 


\section{right thermal > left thermal}
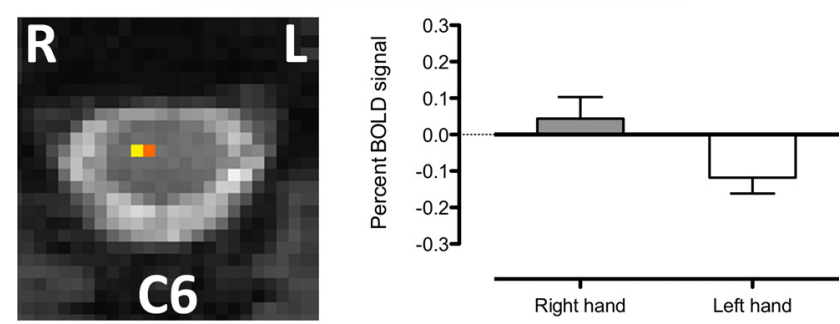

\section{left thermal > right thermal}
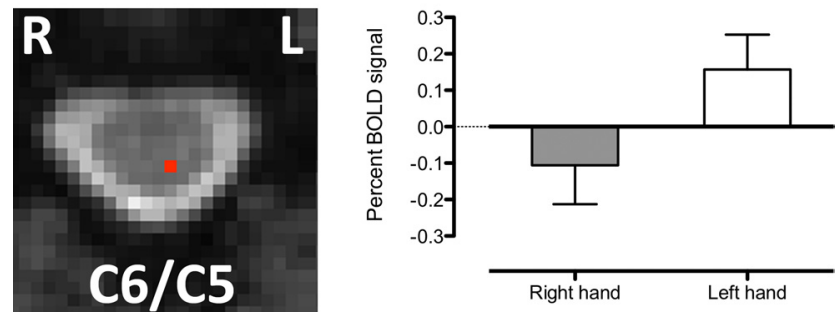

Figure 7. Mixed-effects group differences between LT and RT stimulation. Group differences were observed at single spinal levels for each contrast. The nature of the group difference was investigated by computing the BOLD percentage signal change for the peak voxel (highest Z-score) for each slice and each contrast (bar plots). The bar plots reveal that painful thermal stimulation was associated with positive BOLD signal change ipsilateral to the side of stimulation and with negative BOLD signal change in the contralateral hemicord. The statistical threshold was $p<0.01$ (uncorrected).

Conversely, the more prolonged (3 s duration) thermal stimuli used in this study produced activity at a single spinal level (Fig. 4), which agrees with findings obtained using a contact thermode and 20-s-long painful stimuli applied to the forearm (Eippert et al., 2009). We propose that longer duration thermal stimuli would not produce a coherent reflex response, and that the absence of such effects, in addition to the influence of signal averaging, may explain the apparent specificity of responses obtained with contact thermodes. However, it should be noted that chronic nociceptive stimulation is able to provoke extensive rostrocaudal activation: unilateral pinch in the rat hindpaw produced c-fos immunolabeling over 4-6 and 6-10 segments in the superficial and deeper laminae of the cord, respectively (Hunt et al., 1987; Bullitt, 1991).

In this experiment we observed lateralized spinal cord responses in response to both nonpainful punctate and painful thermal stimuli, with activity ipsilateral to the side of stimulation (Figs. 3-5). Early evidence for a lateralized spinal response measured using blood flow-sensitive techniques comes from autoradiography (Coghill et al., 1991), which found a predominantly ipsilateral response in rat spinal cord following thermal stimulation of the hindpaw. fMRI experiments performed in the axial plane of the anesthetized rat cord have demonstrated increases in blood flow and volume ipsilateral to the side of electrical stimulation (Lilja et al., 2006; Zhao et al., 2009). Immunolabeling with c-fos has demonstrated a clear ipsilateral response following noxious pinch of the rat hindpaw (Bullitt, 1991) and noxious electrical stimulation of the rat forepaw (Lawrence et al., 2004). It is worth noting that $\mathrm{c}$-fos labeling does not provide information about the type of neuronal processing (excitatory or inhibitory) leading to its accumulation. Indeed, following unilateral injec- tion of formalin into the forepaw of the awake rat, increased glucose use was observed in the dorsal horns bilaterally (Porro et al., 1991). However, it is likely that this observation resulted from either behavioral modification (e.g., because of altered locomotion/weight bearing) or inhibitory processing because when the experiment was repeated using anesthetized animals, responses were primarily ipsilateral.

Laterality of spinal cord responses has rarely been studied electrophysiologically (Willis and Coggeshall, 1991) because recording electrodes are typically placed ipsilateral to the stimulated peripheral nerve. In one of the few studies to examine contralateral responses to unilateral stimulation, Fitzgerald (1982) found that a large proportion (54\%) of cells in laminae 4, 5 , and 6 of rat lumbar cord responded to noxious stimulation of the ipsilateral hindlimb but were inhibited following noxious stimulation of the mirror image of the excitatory field (i.e., the opposite hindlimb). It is important to note that in the study by Fitzgerald (1982), animals were decerebrate and spinalized (i.e., the cord was sectioned above the level to be studied), thus ruling out the involvement of descending pain control (Fields, 2004) or diffuse noxious inhibitory control (Le Bars et al., 1979) mechanisms in producing this phenomenon of inhibition or excitation. We investigated the nature of underlying BOLD signal changes giving rise to observed group differences when performing direct pairwise statistical comparison of painful thermal stimulation of the right and left wrists (Fig. 7). It appears that these differences arose because of a simultaneous increase in ipsilateral and reduction in contralateral BOLD signal during noxious thermal stimulation, similar to the pattern of excitation and inhibition observed in the rat spinal cord during noxious stimulation (Fitzgerald, 1982).

We have previously demonstrated that BOLD-sensitive imaging in the human spinal cord is feasible (Brooks et al., 2008; Cohen-Adad et al., 2010) but that care should be taken when interpreting signal change at the cord periphery because this may reflect an increased contribution from large draining veins (Zhao et al., 2008). By limiting our search volume to voxels within the cord and including a physiological noise model when estimating activation, we have attempted to minimize sources of falsepositive activation (Brooks et al., 2008). Others (Stroman et al., 2003; Maieron et al., 2007; Agosta et al., 2008; Summers et al., 2010) did not record or correct for physiological noise but instead relied on low-pass or bandpass filtering of data. Such prefiltering techniques (performed outside the framework of the general linear model) will dramatically increase the number of false positives detected because they can remove a large component of the true variance associated with measurements (Friston et al., 2000).

Concerning the choice of statistical threshold level for assessing spinal cord activation, a recent study acquired resting data to determine likely false-positive detection rates (Summers et al., 2010). However, periods of rest between painful stimuli applied in humans are unlikely to be comparable to "pure" resting conditions. When using fixed intertrial intervals (Summers et al., 2010), the predictability of stimulus onset is likely to give rise to anticipation and preparation processes (Ploghaus et al., 1999; Porro et al., 2002; Ploner et al., 2010), which may result in spinal cord activation. In this study, we report data based on a "corrected" threshold of $p<0.003$, compared with previous spinal fMRI studies that have used an uncorrected threshold of $p<0.01$ (Moffitt et al., 2005; Eippert et al., 2009; Cohen-Adad et al., 2010; Stroman et al., 2011). The chosen threshold is a compromise between the number of statistical tests performed per slice (each containing at most 66 voxels), the likelihood that activation ex- 
tends beyond individual slices (increasing the effective sampling area), and the inadequacy of conventional approaches such as Gaussian random field theory (Worsley et al., 1992) for determining significance levels in such small structures. As a consequence, the data do not meet the strictest criteria set for activation studies of the brain. However, when using nonparametric permutation tests to correct for multiple comparisons, a subset of the results remained significant at $p<0.05$ corrected (voxel-level thresholding), and the pattern of ipsilateral responses to stimulation was confirmed.

Through the combination of significant increases in the temporal signal-to-noise ratio provided by dedicated cervical spinal array coils (Cohen-Adad et al., 2011; Triantafyllou et al., 2011) and techniques to account for physiological noise (Stroman, 2006; Birn et al., 2008; Brooks et al., 2008; Chang et al., 2009), researchers should endeavor to use permutation testing techniques (Nichols and Holmes, 2002) to define corrected thresholds for future group spinal activation studies.

In conclusion, we have developed a method for slice normalization to a template cord, to permit group analysis of spinal cord functional imaging data. By using this technique, we have demonstrated that painful and nonpainful stimuli produce activation in the hemicord ipsilateral to the side of stimulation, in agreement with the known anatomical arrangement of nociceptive input to the cord. By directly comparing thermal stimuli delivered to the right and left arms, we were able to show that painful thermal stimuli produced simultaneously an increased BOLD signal ipsilaterally and a decreased BOLD contralaterally. There is a striking similarity between these observations and the electrophysiological results in rats (Fitzgerald, 1982), which further demonstrates the benefit of noninvasive imaging techniques because they allow sampling of a wider network of neurons.

\section{References}

Agosta F, Valsasina P, Rocca MA, Caputo D, Sala S, Judica E, Stroman PW, Filippi M (2008) Evidence for enhanced functional activity of cervical cord in relapsing multiple sclerosis. Magn Reson Med 59:1035-1042.

Birn RM, Smith MA, Jones TB, Bandettini PA (2008) The respiration response function: the temporal dynamics of fMRI signal fluctuations related to changes in respiration. Neuroimage 40:644-654.

Bouwman CJ, Wilmink JT, Mess WH, Backes WH (2008) Spinal cord functional MRI at 3 T: gradient echo echo-planar imaging versus turbo spin echo. Neuroimage 43:288-296.

Brooks JC, Beckmann CF, Miller KL, Wise RG, Porro CA, Tracey I, Jenkinson M (2008) Physiological noise modelling for spinal functional magnetic resonance imaging studies. Neuroimage 39:680-692.

Brooks JC, Zambreanu L, Godinez A, Craig AD, Tracey I (2005) Somatotopic organisation of the human insula to painful heat studied with high resolution functional imaging. Neuroimage 27:201-209.

Bullitt E (1991) Somatotopy of spinal nociceptive processing. J Comp Neurol 312:279-290.

Chang C, Cunningham JP, Glover GH (2009) Influence of heart rate on the BOLD signal: the cardiac response function. Neuroimage 44:857-869.

Coghill RC, Price DD, Hayes RL, Mayer DJ (1991) Spatial distribution of nociceptive processing in the rat spinal cord. J Neurophysiol 65:133-140.

Cohen-Adad J, Piche M, Rainville P, Benali H, Rossignol S (2007) Impact of realignment on spinal functional MRI time series. Conf Proc IEEE Eng Med Biol Soc 2007:2126-2129.

Cohen-Adad J, Hoge RD, Leblond H, Xie G, Beaudoin G, Song AW, Krueger G, Doyon J, Benali H, Rossignol S (2009) Investigations on spinal cord fMRI of cats under ketamine. Neuroimage 44:328-339.

Cohen-Adad J, Gauthier CJ, Brooks JC, Slessarev M, Han J, Fisher JA, Rossignol S, Hoge RD (2010) BOLD signal responses to controlled hypercapnia in human spinal cord. Neuroimage 50:1074-1084.

Cohen-Adad J, Mareyam A, Keil B, Polimeni JR, Wald LL (2011) 32Channel RF coil optimized for brain and cervical spinal cord at 3 T. Magn Reson Med 66:1198-1208.
Eippert F, Finsterbusch J, Bingel U, Büchel C (2009) Direct evidence for spinal cord involvement in placebo analgesia. Science 326:404.

Fields H (2004) State-dependent opioid control of pain. Nat Rev Neurosci 5:565-575.

Fitzgerald M (1982) The contralateral input to the dorsal horn of the spinal cord in the decerebrate spinal rat. Brain Res 236:275-287.

Friston KJ, Josephs O, Zarahn E, Holmes AP, Rouquette S, Poline J (2000) To smooth or not to smooth? Bias and efficiency in fMRI time-series analysis. Neuroimage 12:196-208.

Hunt SP, Pini A, Evan G (1987) Induction of c-fos-like protein in spinal cord neurons following sensory stimulation. Nature 328:632-634.

Jenkinson M, Bannister P, Brady M, Smith S (2002) Improved optimization for the robust and accurate linear registration and motion correction of brain images. Neuroimage 17:825-841.

Jones AK, Brown WD, Friston KJ, Qi LY, Frackowiak RS (1991) Cortical and subcortical localization of response to pain in man using positron emission tomography. Proc Biol Sci 244:39-44.

Latremoliere A, Woolf CJ (2009) Central sensitization: a generator of pain hypersensitivity by central neural plasticity. J Pain 10:895-926.

Lawrence J, Stroman PW, Bascaramurty S, Jordan LM, Malisza KL (2004) Correlation of functional activation in the rat spinal cord with neuronal activation detected by immunohistochemistry. Neuroimage 22:1802-1807.

Lawrence J, Stroman PW, Malisza KL (2008a) Functional MRI of the cervical spinal cord during noxious and innocuous thermal stimulation in the alpha-chloralose- and halothane-anesthetized rat. Magn Reson Imaging 26:1-10.

Lawrence JM, Stroman PW, Kollias SS (2008b) Functional magnetic resonance imaging of the human spinal cord during vibration stimulation of different dermatomes. Neuroradiology 50:273-280.

Le Bars D, Dickenson AH, Besson JM (1979) Diffuse noxious inhibitory controls (DNIC). I. Effects on dorsal horn convergent neurones in the rat. Pain 6:283-304

Lee MC, Zambreanu L, Menon DK, Tracey I (2008) Identifying brain activity specifically related to the maintenance and perceptual consequence of central sensitization in humans. J Neurosci 28:11642-11649.

Lilja J, Endo T, Hofstetter C, Westman E, Young J, Olson L, Spenger C (2006) Blood oxygenation level-dependent visualization of synaptic relay stations of sensory pathways along the neuroaxis in response to graded sensory stimulation of a limb. J Neurosci 26:6330-6336.

Maieron M, Iannetti GD, Bodurka J, Tracey I, Bandettini PA, Porro CA (2007) Functional responses in the human spinal cord during willed motor actions: evidence for side- and rate-dependent activity. J Neurosci 27:4182-4190.

Merskey H, Bogduk N (1994) Classification of chronic pain: descriptions of chronic pain syndromes and definitions of pain terms, Ed 2. Seattle: IASP Press.

Miron D, Duncan GH, Bushnell MC (1989) Effects of attention on the intensity and unpleasantness of thermal pain. Pain 39:345-352.

Moffitt MA, Dale BM, Duerk JL, Grill WM (2005) Functional magnetic resonance imaging of the human lumbar spinal cord. J Magn Reson Imaging 21:527-535.

Nichols TE, Holmes AP (2002) Nonparametric permutation tests for functional neuroimaging: a primer with examples. Hum Brain Mapp 15:1-25.

Oldfield RC (1971) The assessment and analysis of handedness: the Edinburgh inventory. Neuropsychologia 9:97-113.

Piché M, Cohen-Adad J, Nejad MK, Perlbarg V, Xie G, Beaudoin G, Benali H, Rainville P (2009) Characterization of cardiac-related noise in fMRI of the cervical spinal cord. Magn Reson Imaging 27:300-310.

Ploghaus A, Tracey I, Gati JS, Clare S, Menon RS, Matthews PM, Rawlins JN (1999) Dissociating pain from its anticipation in the human brain. Science 284:1979-1981.

Ploner M, Lee MC, Wiech K, Bingel U, Tracey I (2010) Prestimulus functional connectivity determines pain perception in humans. Proc Natl Acad Sci U S A 107:355-360.

Porro CA, Cavazzuti M, Galetti A, Sassatelli L, Barbieri GC (1991) Functional activity mapping of the rat spinal cord during formalin-induced noxious stimulation. Neuroscience 41:655-665.

Porro CA, Baraldi P, Pagnoni G, Serafini M, Facchin P, Maieron M, Nichelli P (2002) Does anticipation of pain affect cortical nociceptive systems? J Neurosci 22:3206-3214.

Pórszász R, Beckmann N, Bruttel K, Urban L, Rudin M (1997) Signal 
changes in the spinal cord of the rat after injection of formalin into the hindpaw: characterization using functional magnetic resonance imaging. Proc Natl Acad Sci U S A 94:5034-5039.

Raij TT, Numminen J, Närvänen S, Hiltunen J, Hari R (2005) Brain correlates of subjective reality of physically and psychologically induced pain. Proc Natl Acad Sci U S A 102:2147-2151.

Rainville P, Duncan GH, Price DD, Carrier B, Bushnell MC (1997) Pain affect encoded in human anterior cingulate but not somatosensory cortex. Science 277:968-971.

Sarlani E, Farooq N, Greenspan JD (2003) Gender and laterality differences in thermosensation throughout the perceptible range. Pain 106:9-18

Stroman PW (2006) Discrimination of errors from neuronal activity in functional MRI of the human spinal cord by means of general linear model analysis. Magn Reson Med 56:452-456.

Stroman PW, Tomanek B, Krause V, Frankenstein UN, Malisza KL (2003) Functional magnetic resonance imaging of the human brain based on signal enhancement by extravascular protons (SEEP fMRI). Magn Reson Med 49:433-439.

Stroman PW, Coe BC, Munoz DP (2011) Influence of attention focus on neural activity in the human spinal cord during thermal sensory stimulation. Magn Reson Imaging 29:9-18.

Summers PE, Ferraro D, Duzzi D, Lui F, Iannetti GD, Porro CA (2010) A quantitative comparison of BOLD fMRI responses to noxious and innocuous stimuli in the human spinal cord. Neuroimage 50:1408-1415.

Talbot JD, Marrett S, Evans AC, Meyer E, Bushnell MC, Duncan GH (1991)
Multiple representations of pain in human cerebral cortex. Science 251:1355-1358.

Triantafyllou C, Polimeni JR, Wald LL (2011) Physiological noise and signal-to-noise ratio in fMRI with multi-channel array coils. Neuroimage 55:597-606.

Willis WD, Coggeshall RE (1991) Sensory mechanisms of the spinal cord, Ed 2. New York: Plenum.

Woolf CJ, Salter MW (2000) Neuronal plasticity: increasing the gain in pain. Science 288:1765-1769.

Woolrich MW, Behrens TE, Beckmann CF, Jenkinson M, Smith SM (2004) Multilevel linear modelling for FMRI group analysis using Bayesian inference. Neuroimage 21:1732-1747.

World Medical Association (1996) Declaration of Helsinki. Br Med J 313: 1448.2 (http://www.bmj.com/content/313/7070/1448.2).

Worsley KJ, Evans AC, Marrett S, Neelin P (1992) A three-dimensional statistical analysis for CBF activation studies in human brain. J Cereb Blood Flow Metab 12:900-918.

Zhao F, Williams M, Meng X, Welsh DC, Coimbra A, Crown ED, Cook JJ, Urban MO, Hargreaves R, Williams DS (2008) BOLD and blood volume-weighted fMRI of rat lumbar spinal cord during non-noxious and noxious electrical hindpaw stimulation. Neuroimage 40:133-147.

Zhao F, Williams M, Meng X, Welsh DC, Grachev ID, Hargreaves R, Williams DS (2009) Pain fMRI in rat cervical spinal cord: an echo planar imaging evaluation of sensitivity of BOLD and blood volume-weighted fMRI. Neuroimage 44:349-362. 